\title{
Movimientos feministas en México y sus transformaciones en el contexto de la pandemia por la COVID-19 a partir de las prácticas comunicativas en las redes sociodigitales
}

Feminist Movements in Mexico and Their Transformations in the Context of the COVID-19 Pandemic Based on Communicative Practices in Social Network Sites

Doctora en Comunicación y Estudios Culturales por la Nottingham Trent University (Reino Unido), y maestra en Comunicación de la Ciencia y la Cultura por el ITESO, Universidad Jesuita de Guadalajara (México). Es miembro del Sistema Nacional de Investigadores (SNI) del Conacyt, de la Asociación Mexicana de Investigadores en Comunicación (AMIC) y de la Asociación Latinoamericana de Investigadores en la Comunicación (ALAIC). Desde hace 17 años, se especializa en cultura digital, movimientos sociales y metodología cualitativa digital. 



\title{
Movimientos feministas en México y sus transformaciones en el contexto de la pandemia por la COVID-19 a partir de las prácticas comunicativas en las redes sociodigitales ${ }^{1}$ \\ Feminist Movements in Mexico and Their Transformations in the Context of the COVID-19 Pandemic Based on Communicative Practices in Social Network Sites
}

\author{
Lidia Ángeles García González \\ Universidad de Guanajuato, México \\ la.garciagonzalez@ugto.mx (https://orcid.org/oooo-0001-9363-5280) \\ Recibido: 26-07-2021 / Aceptado: 12-10-2021 \\ https://doi.org/10.18800/conexion.202102.007
}

\section{PALABRAS CLAVE / KEYWORDS}

Activismo digital feminista, COVID-19, México, redes sociodigitales / digital feminist activism, COVID-19, Mexico, social network sites

\section{RESUMEN}

El presente artículo es un estudio de corte cualitativo sobre los movimientos feministas en México y sus transformaciones en el contexto de la pandemia por la COVID-19 a partir de las prácticas comunicativas en las redes sociodigitales. Su propósito es conocer los desafíos, los cambios y las estrategias comunicativas empleadas ante el confinamiento, que limitó el uso del espacio público para la protesta feminista y el acompañamiento a mujeres víctimas de la violencia. Asimismo, presenta una discusión teórica sobre los movimientos sociales y la acción colectiva feminista en el contexto de la pandemia ocasionada por la COVID-19 (Della Porta, 2020b; Pleyers, 2021; Tabbush, 2021). Los resultados evidencian cambios significativos en sus estrategias comunicativas en las redes sociodigitales y un incremento en el agotamiento emocional, dado el confinamiento impuesto por la contingencia sanitaria; sin embargo, el empleo de iniciativas locales muestra una perspectiva esperanzadora de volver a retomar las calles en el futuro.

\section{ABSTRACT}

This article is a qualitative study on feminist movements in Mexico and their trans-

\footnotetext{
${ }^{1}$ Agradecimientos: agradezco al Consejo Nacional de Ciencia y Tecnología (Conacyt) por su apoyo para la Estancia Posdoctoral por México 2021, y al Sistema Nacional de Investigadores (SNI), estancia que se realiza en la Universidad de Guanajuato, en la maestría de Análisis Político del Departamento de Estudios Políticos y Gobierno, bajo la asesoría de la Dra. Ericka López Sánchez.
} 
formations in the context of the COVID-19 pandemic based on communicative practices in social network sites. Its purpose is to learn about the challenges, changes and communicative strategies employed in the face of the confinement, which limited the use of public space for feminist protest and accompaniment of women victims of violence. It also presents a theoretical discussion on social movements and feminist collective action in the context of the COVID-19 pandemic (Della Porta, 2020b; Pleyers, 2021; Tabbush, 2021). The results show significant changes in their communicative strategies in social network sites and an increase in emotional exhaustion, given the confinement imposed by the health contingency; however, the use of local initiatives shows a hopeful perspective of retaking the streets in the future.

\section{Movimientos feministas en México y sus transformaciones en el contexto de la pandemia por la COVID-19 a partir de las prácticas comunicativas en las redes sociodigitales}

La persistencia de los feminicidios exhibe que las acciones siguen siendo escasas para prevenir, atender y sancionar la violencia contra las mujeres en México, que se encuentra situada en un contexto de violencia generalizada, discriminación e impunidad. Marzo de 2021 ha sido considerado el mes más violento para las mujeres en México desde el año 2015: en
359 muertes violentas; esto se traduce en 11 feminicidios al día. Asimismo, hubo un incremento del $19.2 \%-13317$ en totalde presuntos delitos de violencia familiar y en todas sus modalidales subieron un $18 \%-208$ casos más- de enero-abril 2020 a enero-abril 2021; en el mismo periodo, los presuntos delitos de violación aumentaron $21.4 \%$-es decir, 1175 más casos- (Secretariado Ejecutivo del Sistema Nacional de Seguridad Pública, 2021a, 2021b).

En el periodo entre el año 2018 y el 20 de junio de 2021, el Registro Nacional de Personas Desaparecidas y No Localizadas (2021) documentó la desaparición de $25340 \mathrm{mu}$ jeres, niñas y adolescentes en México. De la misma manera, el Secretariado Ejecutivo del Sistema Nacional de Seguridad Pública (2020) indicó que, de enero a diciembre de 2020, en promedio, 10.2 mujeres y niñas fueron asesinadas al día en México. $\mathrm{El}$ año 2020 cerró con el mayor número de denuncias por violencia familiar desde que se tiene registro: 220028 (Secretariado Ejecutivo del Sistema Nacional de Seguridad Pública, 2020).

Finalmente, el panorama para las mujeres en México es desalentador, ya que el Gobierno federal recortó 37583000 pesos al presupuesto asignado a la Alerta de Violencia de Género contra las Mujeres en los lugares que más feminicidios registraron en el año 2020: Estado de México, Veracruz, Nuevo León, Ciudad de México, Puebla, Jalisco, Nayarit y Zacatecas («El 
Gobierno de México está desarmando los mecanismos de protección a la mujer», 2020, párr. 2). Y, en ese mismo año, el Gobierno recortó 1872 millones de pesos de 20 programas de apoyo a las mujeres (2020, párr. 6). En este sentido, la inacción del Gobierno ante este panorama ha demostrado cómo la violencia contra las mujeres es reproducida por el mismo Estado responsable de protegerlas. En consecuencia, las mismas mujeres mexicanas han decidido convertirse en sus propias protectoras. Las colectivas feministas han creado redes de apoyo para las víctimas de la violencia doméstica cuya situación empeora por las restricciones de la contingencia sanitaria (Ventura Alfaro, 2020). Por ello, la pregunta que guía esta investigación es la siguiente: ¿de qué manera se ha transformado el activismo digital feminista en México ante el contexto de la pandemia por la COVID-19?

\section{Los movimientos sociales y la acción colectiva feminista en el contexto de la pandemia causada por la COVID-19}

Los movimientos sociales, ante la emergencia de la pandemia por la COVID-19, se vieron intensamente perjudicados por la necesidad de millones de mujeres y hombres de tener que quedarse en casa. Según (Pleyers, 2020), esta situación dio un alto a la ola de protestas ciudadanas masivas en diversas partes del mundo por la democracia y la justicia social en 2019. No obstante, como afirma Pleyers, los movimientos por la justicia social se adaptaron a las circunstancias inesperadas y fueron realmente muy activos en este difícil periodo (2020, p. 307).

La contingencia a raíz de la pandemia por la COVID-19 ha representado grandes retos para los movimientos sociales en todo el mundo. Della Porta indica que, en las fases iniciales de la pandemia, se han encontrado con lo que los medios de comunicación y los activistas pueden ver como una nueva ola de protesta (2020a, p. 355). La acción colectiva ante el miedo al contagio y la medida de bloqueo que impedía rotundamente la movilización en el espacio físico aparentemente pusieron en riesgo las accciones colectivas; ante esta situación, los activistas crearon diversas formas de manifestar el incremento de demandas, lo que dio paso a nuevas tácticas y repertorios de acción colectiva, como señala Della Porta: las caravanas, los cacerolazos, la interpretación colectiva de canciones de protesta desde los balcones, las acciones transmitidas en directo, las concentraciones digitales, las marchas virtuales, los paros, los boicots y las huelgas de alquiler se han multiplicado como formas de denunciar lo que la pandemia hizo más evidente y menos tolerable: la profundidad de las desigualdades y sus dramáticas consecuencias en vidas humanas (2020a, p. 355).

Asimismo, las activistas han producido nuevos conocimientos utilizando recursos digitales para el intercambio de saberes y la enseñanza en línea; han contri- 
buido a conectar los diferentes campos de conocimiento que la hiperespecialización de la ciencia tiende a fragmentar (Della Porta, 2020a, p. 356). El valor central que han difundido, lejos de una conceptualización desde la caridad, ha sido la solidaridad (Ventura Alfaro, 2020), que combate el individualismo exacerbado proveniente del sistema económico neoliberal. Los activistas actuaron, como señala Pleyers, a partir de dedicar tiempo y esfuerzos a iniciativas locales de solidaridad: «Las redes de ayuda mutua son probablemente las que más energía han movilizado» (2021, p. 9).

Además, mediante el uso de plataformas digitales, se pueden llevar a cabo protestas en línea, «entre las que se incluyen, entre otras, las peticiones electrónicas que se han multiplicado en este período» (Della Porta, 202ob, p. 176). Ante todo, los movimientos sociales, lejos de desactivarse por el confinamiento y las dificultades de la pandemia, se mantuvieron activos desde antes de la pandemia (Pleyers, 2021). Este último autor indica que, en esta situación de la pandemia causada por el coronavirus, los movimientos sociales enfocaron sus actividades en satisfacer necesidades inmediatas. Estas iniciativas fueron consideradas a veces «como "despolitizadoras" puesto que desvían la energía de los militantes y las organizaciones de las demandas de cambio estructural en favor de prácticas que solo compensan las deficiencias del siste-
En el caso particular del movimiento feminista durante la pandemia por la COVID-19, se ha mantenido activo y ha señalado el incremento de la violencia doméstica durante el confinamiento: las activistas feministas denunciaron un aumento de la violencia doméstica (Al-Ali, 2020, p. 335), y demandan al Estado sobre esta catastrófica situación para proveer de alojamientos solidarios a las mujeres víctimas; fueron las colectivas feministas autogestionadas quienes expandieron robustamente sus redes de solidaridad en todo México «para hacer frente a las consecuencias socioeconómicas más graves del virus a nivel local: escasez de alimentos, medicinas y otros productos esenciales, en medio del aumento de la violencia en el interior de los hogares (Ventura Alfaro 2020)» (Pleyers, 2021, pp. 10-11). Las activistas feministas proponen un modelo de sociedad en el que el cuidado es parte esencial (Suárez-Krabbe, 2020).

Según Ventura Alfaro (2020, p. 87), el movimiento feminista se distingue por dos características representativas de la cuarta ola feminista: que está basado en una epistemología feminista inclusiva e interseccional, y que utiliza las plataformas sociales y la web como su principal herramienta organizativa, ahora acentuada por la cuarentena. No obstante, frente a la problemática sanitaria, económica, emocional y social, las colectivas feministas mexicanas continuaron incrementando su trabajo. En este sentido, los repertorios de acción colectiva feminista compren- 
den, como apunta Ventura Alfaro, talleres en línea, grupos de lectura y seminarios, que son organizados semanalmente por diferentes organizaciones para continuar el debate sobre la violencia, el acoso sexual, las condiciones de trabajo, los estereotipos de género, los derechos reproductivos y muchos otros temas que afectan a las mujeres en su vida cotidiana (2020, p. 85).

Otra de las características que tienen estas redes feministas es que no pertenecen a organismos gubernamentales $u$ organizaciones no gubernamentales, y mantienen una relación muy cercana de contacto con las víctimas de la violencia doméstica. Las organizaciones de la sociedad civil, los grupos de derechos de la mujer y las organizaciones feministas jóvenes se readaptaron para convertirse en proveedores de servicios en las comunidades, porque el Estado no era capaz de prestarlos en la escala requerida.

Un momento crucial durante la pandemia por la COVID-19 ha sido la reducción de fondos, lo que dio paso a que los movimientos se organizaran de forma diferente para responder a la pandemia: nació un medio de movilización totalmente nuevo (Rodriguez, 2021). Cuando inició el confinamiento y las autoridades gubernamentales dieron la instrucción de quedarse en casa, diversas colectivas feministas comenzaron a organizar campañas de acompañamiento psicológico y a brindar espacios como refugios para aquellas que vivían en entornos violentos, de modo que no se presentaran mayores agresiones durante la cuarentena (Badillo, 2020).

En México, la instrucción \#QuédateEnCasa evidenció que el aumento de la violencia de género es resultado de la falta de atención e ineficiente acceso de las mujeres a la justicia. Para la autora Núñez Cetina (2021), el Estado mexicano, ante la emergencia sanitaria, debió proveer de un presupuesto y de apoyo humano, dados los efectos de la pandemia, que «al interior de los hogares también demandaba[n] que las medidas de control punitivo se reforzaran o, por lo menos, se mantuvieran para frenar la violencia hacia las mujeres y las niñas, la cual en muchos casos es letal» (Núñez Cetina, 2021, p. 119).

Bajo el contexto de la pandemia por la COVID-19, una diversidad de movimientos de mujeres y movimientos territoriales actuaron como estabilizador informal de este «triple shock»; llegaron a lugares donde el Estado no lo hace y compensaron la escasa ayuda asistencial. Esto se debió, en gran medida, al trabajo comunitario de mujeres pobres, periféricas o afrodescendientes, que se organizaron en comedores populares y servicios de cuidado comunitarios. Se asistió a víctimas de violencia de género, supliendo también la desinformación, compensando en parte deficiencias estatales y abogando por una mayor justicia de género, social y racial. Sin embargo, para Tabbush (2021): 
Esta red informal de protección es frágil y no puede compensar la ausencia de políticas públicas. Para que la pandemia verdaderamente se vuelva una oportunidad de transformación, se deben escuchar estas voces en la construcción de un Estado social activo [...].

Las demandas y la imaginación política de feministas y de movimientos sociales promueven un orden global más justo y un contrato social más igualitario (pp. 104-105).

En el artículo «Efectos de la pandemia por la Covid-19 en las movilizaciones feministas de la Ciudad de México», de Portillo Sánchez y Beltrán Fuentes (2021), las autoras concluyen que las movilizaciones feministas se caracterizan por construir «redes de protección y cuidado». Identifican una serie de estrategias para mantener el resguardo de «las integrantes de colectivas tanto en los espacios físicos como digitales» (Portillo Sánchez y Beltrán Fuentes, 2021, p. 30); por otro lado, en el contexto de la pandemia por la COVID-19, la seguridad digital con perspectiva de género se ha convertido en un tema prioritario al que se le buscan soluciones.

Además, el activismo digital feminista es, en sí mismo, una actividad con un alto «agotamiento emocional» que, bajo el contexto del confinamiento, se ha incrementado (Portillo Sánchez y Beltrán que una de las prácticas que se han implementado al interior de las colectivas ha sido «la vigilancia con ellas mismas y con sus compañeras» (p. 30).

Para Mendes et al. (2019, p. 74), el activismo digital feminista puede ser altamente explotador y, en muchas ocasiones, no tiene compensación económica. En cuanto a las actividades que realizan en los sitios web, el trabajo requiere mucho tiempo y con frecuencia suele ser muy tedioso y - como se ha indicado anteriormente- emocionalmente agotador; en consecuencia, las activistas han optado por determinadas prácticas para lidiar con el agotamiento, que incluyen, como apuntan las autoras, tocar música, la meditación, el yoga, la lectura de obras feministas, los paseos por el parque, la participación en comunidades feministas, y tomarse un descanso de su activismo o de sus actividades en línea (Mendes et al., 2019, p. 97).

A pesar de todas las desventajas e implicaciones negativas que han enfrentado las colectivas feministas durante la pandemia ocasionada por la COVID-19, existen redes que están impulsando una respuesta sin precedentes en este contexto, desde intervenciones políticas multilaterales hasta acciones solidarias en barrios urbanos de bajos ingresos; las habilidades técnicas y las conexiones a largo plazo han permitido a las feministas, dentro y fuera de los Gobiernos, ejercer rápidamente presión sobre los responsables po- 
líticos nacionales e internacionales. Tabbush y Friedman (2021, p. 637) sostienen que el poder de la cuarta ola sigue impulsando los derechos de la mujer y la justicia de género, y es posible que vuelvan a inundar las calles, lo que da cuenta de una visión esperanzadora del activismo feminista en el contexto de la pandemia actual por la COVID-19.

\section{Metodología}

Este artículo tiene como objetivo examinar las dimensiones de la apropiación de los espacios digitales para la acción colectiva por parte de coaliciones feministas mediante los discursos que articulan contra la violencia de género, y conocer cuáles son los repertorios de acción colectiva digital empleados. En tal sentido, se opta por el uso de las entrevistas cualitativas, que pueden ser una ventana al mundo cotidiano de los activistas y que generan representaciones que encarnan las voces de los sujetos, minimizando -al menos en la medida de lo posible - la voz del investigador (Ragin y Amoroso, 1994). Para Klandermans y Staggenborg (2002, pp. 95-96), utilizar entrevistas para investigar los movimientos sociales ofrece la oportunidad de conocer el contexto de las motivaciones, creencias y actitudes de los participantes en los movimientos sociales. Además, permite investigar al individuo y sus visiones colectivas, imaginaciones, esperanzas, expectativas, críticas del presente y proyecciones del futuro, sobre las que descansa la posibilidad de la ac- ción colectiva y a través de las cuales los movimientos sociales se forman, resisten o se dispersan (2002, pp. 95-96).

James y Busher (2012) indican que las plataformas para videollamadas pueden usarse con propósitos de investigación, y también subrayan cómo los sitios de redes sociales como Facebook están siendo explorados ahora como un lugar donde se pueden realizar entrevistas en vivo, en lugar de enviar preguntas por correo electrónico; debe considerarse además que actualmente, ante el contexto de la pandemia global por la COVID-19, plataformas como Zoom, Google Meet, Jitsi Meet y Teams proporcionan diferentes posibilidades para llevar a cabo entrevistas en línea, con la ventaja adicional de que las sesiones se pueden grabar.

Para este estudio, se realizaron, entre noviembre de 2020 y febrero de 2021, 14 entrevistas con activistas feministas de nueve colectivas mexicanas. El muestreo se construyó en un universo de 35 colectivas feministas en México, que se contactaron vía correo electrónico. Previamente, se obtuvo un correo de contacto en sus páginas de Facebook. Cada entrevista duró entre 40 minutos y una hora y media, y se realizó y grabó por videollamada mediante la plataforma Google Meet. Posteriormente, se les pidió a las activistas mantener comunicación vía WhatsApp o correo electrónico por si hubiera alguna pregunta o duda sobre la entrevista. 
Tabla 1

Información sobre las activistas entrevistadas

\begin{tabular}{|c|c|c|c|c|c|}
\hline & $\begin{array}{c}\text { Nombre de la colectiva y } \\
\text { activista }\end{array}$ & $\begin{array}{l}\text { Año de } \\
\text { formación }\end{array}$ & Profesión & $\begin{array}{l}\text { Lugar de } \\
\text { residencia }\end{array}$ & Edad \\
\hline 1 & $\begin{array}{l}\text { Las del Aquelarre Feminista } \\
\text { (Ana Elena Contreras) }\end{array}$ & 2016 & Abogada & $\operatorname{CDMX}$ & 44 \\
\hline 2 & $\begin{array}{l}\text { Colectiva Artemisas } \\
\text { Veterinarias (Anónimas) }\end{array}$ & 2020 & $\begin{array}{l}\text { Estudiantas } \\
\text { médica } \\
\text { veterinaria }\end{array}$ & CDMX & $\begin{array}{l}34,21,19 \\
21,21\end{array}$ \\
\hline 3 & $\begin{array}{l}\text { Nosotras Colectivas } \\
\text { (Magaly Ortiz) }\end{array}$ & 2015 & Lic. en Sociología & $\begin{array}{l}\text { Hermosillo, } \\
\text { Sonora }\end{array}$ & 26 \\
\hline 4 & $\begin{array}{l}\text { Siempre Unidas } \\
\text { (Tania Ramírez) }\end{array}$ & 2017 & Lic. en Lenguas & $\begin{array}{c}\text { Cancún, } \\
\text { Quintana Roo }\end{array}$ & 41 \\
\hline 5 & $\begin{array}{l}\text { Luchadoras } \\
\text { (Lulú Barrera) }\end{array}$ & 2012 & $\begin{array}{l}\text { Lic. en Letras } \\
\text { Españolas }\end{array}$ & $\operatorname{CDMX}$ & 39 \\
\hline 6 & $\begin{array}{l}\text { Abogadas con Glitter } \\
\text { (Anónimas) }\end{array}$ & 2019 & $\begin{array}{l}\text { Estudiantas de } \\
\text { Derecho }\end{array}$ & $\operatorname{CDMX}$ & $26,23,28,28$ \\
\hline 7 & $\begin{array}{l}\text { Restauradoras con Glitter } \\
\text { (Sofía Riojas) }\end{array}$ & 2019 & Arquitecta & $\operatorname{CDMX}$ & 33 \\
\hline
\end{tabular}

Nota. Esta tabla muestra la lista de las activistas feministas entrevistadas.

\section{Transformaciones del activismo digital feminista en México a partir de la pandemia por la COVID-19}

Uno de los primeros cambios que derivaron de la pandemia por la COVID-19 dentro de las colectivas feministas en México está relacionado con el cambio de las dinámicas de trabajo al interior de cada colectiva. Lo central consistía en mantener, primero, la salud física y la integridad emocional de cada una, dadas las consecuencias del encierro; no obstante, por el hecho de trabajar en casa, existió una exigencia por producir durante el confinamiento, lo que derivó en una resistencia. Ana Elena, de la colectiva Las del Aquela-
Al principio, en la pandemia todo el mundo entró en una psicosis de «tenemos que producir y tenemos que hacernos presentes». Yo les comentaba a mis compañeras: la verdad, no me siento en la necesidad ni de producir ni de estar presente. Entonces nosotras, todas, agarramos ese camino de estar como muy aletargadas [...], porque también sentía yo que era una demanda muy patriarcal. Sí se modificó: por ejemplo, nosotras estuvimos teniendo menos reuniones en todo el periodo de lo que va de la pandemia [...]. No hemos podido sacar como otras campañas en este periodo, porque también estamos como muy ocupadas en en el rollo de la 
sobrevivencia [...]. Aun así, nos aventamos a sacar la Marcha de las Catrinas ${ }^{2}$, que hacemos cada año; este, con todos los temores que había por lo del COVID-19, decidimos salir porque también pensamos y dijimos que no salir era, pues, justo como ceder el espacio. Es importante decir que, a pesar de la pandemia, a las mujeres y a las niñas nos siguen matando.

A pesar de que la acción colectiva feminista se movió a los espacios digitales, el traslado de las actividades a la virtualidad fue paulatino; es decir, el gran reto que tuvieron fue que, durante el confinamiento, el espacio se redujo a un solo lugar -el hogar- y la apropiación de las plataformas digitales por parte de las activistas fue un proceso gradual y complejo, en el que se vieron en la necesidad de desarrollar sus habilidades para crear contenidos digitales. Una activista de la colectiva Artemisas Veterinarias lo manifiesta así:

Trasladamos todo a la virtualidad, porque anteriormente llevábamos todo de manera presencial, y nos veíamos, comíamos juntas y platicábamos cosas de la colectiva. Ahora todo es por este medio. El año pasado se hicieron acciones del \#25N en la facultad y ahorita lo trasladamos a hacer un conversatorio en línea, película. Es mucho trabajo. Es más complicado, porque tienes el trabajo de las compañeras, que están en la facultad, la escuela, la casa y la colectiva: todo está como en el mismo espacio. A mí me pasa igual en mi trabajo, la escuela, la casa y la colectiva, y se hizo más fuerte esta situación de la pandemia, que nos está afectando mucho, sobre todas las mujeres, y en la colectiva no es la excepción. Y la parte de digitalizar todo ha sido compleja y un trabajo arduo, porque venimos de hacer muchas cosas fuera de la virtualidad.

Otro de los cambios de las colectivas feministas fue la reducción de activistas en su organización debido a la pandemia por la COVID-19. En el caso de Nosotras Colectiva, de la ciudad de Hermosillo, Sonora, la colectiva se limitó en sus actividades; sin embargo, durante la pandemia, retomaron la producción de un pódcast con las herramientas digitales que internet les proporcionó en el espacio digital. Como indica Piñeiro-Otero, «colectividades y personas individuales se han lanzado a la creación de diferentes tipos de contenidos, que van desde temáticas más próximas a la teoría y praxis feministas, a la presentación de voces y perspectivas más diversas y disidentes» (2021, p. 234). Esta información, la mayoría de las veces, es diseñada y producida por ellas mismas, o bien se apoyan en una diseñadora o diseñador. Estos sitios de redes sociales se 
han convertido en muy relevantes para estas mujeres:

Pues ahorita, antes de la pandemia, éramos más y se redujo el tamaño de la colectiva y entonces eso nos afectó y luego, pues, inmediatamente llegó la pandemia y hemos estado un poco inactivas [...], y no nos hemos visto mucho en persona. Estamos ideando formas de seguir con la colectiva, de seguir organizando cosas así durante la pandemia y estábamos pensando en hacer un taller de aborto que fuera virtual (Nosotras Colectiva).

Una de las desventajas que enfrentaron las activistas feministas ante la contigencia sanitaria en México fue la imposibilidad de conocer personalmente a las mujeres víctimas a quienes estaban acompañando, lo cual les generó dudas sobre si las personas que solicitaban ayuda realmente se encontraban en situación de vulnerabilidad. Tania Ramírez, de la colectiva quintanarroense Siempre Unidas, argumenta:

Como que todo es virtual, antes, pues, por ejemplo en la pandemia, hay víctimas que yo no conocí que me hubiera gustado conocer en persona que no les pudimos dar el acompañamiento como me hubiera gustado, porque, pues, también por ejemplo nos llegaban casos de mujeres violentadas que necesitan apoyo económico, que quién era. Y qué bueno el apoyo brindado, pero me hubiera gustado también hacer el acompañamiento. Yo no sé si llegó al hogar donde tenía que llegar o [si] era el adecuado, porque muchas veces pedimos apoyo económico para estos casos y si nos ayudan no [es] nada más a través de nuestras páginas, sino a través de nuestras redes de mujeres, pero muchas veces ya no sabemos a quién está llegando la ayuda. A mí me gusta conocer a las personas. Nos ha limitado mucho la pandemia y espero que pase pronto, porque la para presencial es muy importante para mí.

A pesar de las adversidades que las colectivas feministas mexicanas han tenido que enfrentar a raíz de la pandemia por la COVID-19, la situación coincide con la perspectiva de Tabbush y Friedman (2021), quienes sostienen que el movimiento feminista sigue avivando la lucha por los derechos de la mujer y la justicia de género. La activista Lulú Barrera, de la colectiva Luchadoras, lo exterioriza así:

Yo creo que el feminismo en México está vivo y está pasando por los mejores momentos a pesar de la pandemia, y que sigue ahí. O sea, es una fuerza, una latencia que es como una bola de nieve que empezó en \#24A de 2016 y que sigue hasta ahora, y que cada vez se expande más y más y que no va a parar. Lo veo muy claro: no va a parar. 
Las marchas del 8 de marzo a lo largo del país y del posterior paro nacional de mujeres y niñas del \#9M en 2020 en México fueron históricas; fueron, asimismo, dos grandes esfuerzos para visibilizar las luchas de las mujeres y el feminismo en México, lo que dejó aprendizajes para las activistas sobre el poder de las mujeres a partir de la acción colectiva y la democratización de la lucha por la defensa de los derechos de las mujeres en México y la justicia. Una activista de la colectiva Abogadas con Glitter lo describe así:

Yo pienso que también se debe a la marcha del 8 de marzo junto con el paro nacional de mujeres. Eso fue muy impactante en la vida de muchas personas; fue una marcha en donde aprendimos mucho y donde se vio muchísima gente, mujeres, niñas, ancianas [para las] que fue su primera marcha. Se pudo ver más gente apoyando el movimiento feminista, más mujeres. Y después de eso llega el confinamiento y te quedas con esa idea de [que] sí existe la violencia, es real, y muchas mujeres salieron a manifestarse el \#8M. Entonces llega que te encierran con tu agresor y empiezas a visualizar tu violencia en tu casa. Las llamadas al 911 se dispararon. El Estado lamentablemente negó la situación y se genera un movimiento, \#NosotrasTenemosOtrosDatos. Entonces, eso hizo que se viera más y que sí existe la violencia y que lamentablemente muchas de ellas viven con su agresor, y también eso hizo que más personas mujeres se denominaran feministas.

Sin embargo, existen visiones opuestas sobre la transformación del activismo feminista en el contexto de la pandemia por la COVID-19, porque, desde la perspectiva de la colectiva Restauradoras con Glitter, la potencia del movimiento feminista en México radica en la toma del espacio público, que se vio limitada por las restricciones al espacio público por la contingencia sanitaria. La activista Sofía Riojas lo argumenta así:

Es que la toma del espacio público era lo que estaba potenciando la lucha feminista. O sea, nunca habíamos tenido marchas multitudinarias del \#8M, nunca, y justo después del \#8M del año pasado que pasó en la pandemia y la actividad y las cosas que estaban sucediendo se frenaron por completo. Entonces creo que hay otro tipo de dinámicas, como que tardamos además mucho tiempo en reponernos de ese bajón. Pero definitivamente no creo que haya potenciado nada. O sea, lo que estaba antes era muy potente [...]. Para mí ha sido como un superbajón, porque ha frenado muchas de las cosas que ya estamos pudiendo echar a andar presencialmente y creo que compartir la toma de las calles con las compañeras era fundamental; de pronto ahora hay discusiones en Twitter o asî de Feministlán, que no son 
las que corresponden a la realidad; o sea, son como discusiones desde el escritorio y estar en la calle con las compañeras era una cosa.

Esta perspectiva se deriva de las características y objetivos que tiene esta colectiva feminista, ya que surge como una respuesta ante la criminalización de la protesta en los medios de comunicación y por determinados actores políticos, en específico sobre las expresiones realizadas en la superficie de la base de la Columna de la Independencia en la Ciudad de México el 16 de agosto de 2019 (Restauradoras con Glitter, 2019, párr. 1).

Parte de su quehacer ha consistido en el acceso concedido a través de las autoridades del Gobierno al basamento de la Columna de la Independencia, en donde han realizado un registro minucioso de las pintas con diferentes técnicas de fotografías, dibujos, drones y modelos 3D, todo esto con la finalidad de hacer un catálogo digital en el cual estas pintas estén georreferenciadas y con un pie de nota explicativo en cada caso. Asimismo, el propósito de este registro es que sea utilizado por otros grupos y que este trabajo sea tratado como una memoria del movimiento mexicano contemporáneo de mujeres.

Es decir, una de sus actividades principales se ubica en el espacio público y las calles; de ahí podría interpretarse la críti- en la academia como clictivismo, que se refiere al uso de las redes sociodigitales para promover una causa. Es importante mencionar que, a lo largo de la pandemia, el trabajo de las colectivas se ha intensificado mediante el uso de las redes sociodigitales; sin embargo, en el presente estudio, no se ubicó una tensión de fondo entre las actividades de las colectivas feministas en el espacio digital frente a la apropiación del espacio público, ya que consideran que todo tipo de acción colectiva es válida. Lo que sí es esencial para la acción colectiva de las activistas feministas es que la toma del espacio público y la calle antes de la pandemia estaba potenciando y democratizando de manera masiva su lucha, y visibilizando el descontento creciente por la ola de violencia contra las mujeres en México.

\section{Discusión y conclusiones}

La pandemia por la COVID-19 representó un reto para las activistas feministas en todo el mundo, sin excepción alguna. El movimiento feminista en México se vio frente a una diversidad de desafíos, desde adoptar nuevos repertorios de acción colectiva -principalmente en el espacio digital, lo que implicó adquirir nuevos conocimientos sobre el manejo de herramientas digitales- hasta implementar iniciativas locales de solidaridad tomando el papel del Estado y expandiendo, a la vez, sus redes de apoyo.

En la médula de todas estas iniciativas 
se encuentran las redes de autocuidado, apoyo y contacto con las víctimas, dado el incremento de la violencia, así como la creación de campañas de acompañamiento como medida compensatoria ante las deficiencias del Estado mexicano. Del mismo modo, por el encierro en casa derivado de la pandemia por la COVID-19, se gestó la conformación de nuevas redes de apoyo entre mujeres y la creación de nuevas colectivas feministas en México; esto ocurrió a partir de la contingencia vivida por muchas mujeres que se vieron forzadas a convivir con su agresor las 24 horas del día y todos los días.

A partir del presente estudio, pudimos comprobar que las colectivas feministas mexicanas entrevistadas tuvieron que enfrentar diversos retos ante la exigencia patriarcal de producir en el confinamiento; sin embargo, algunas activistas feministas crearon una resistencia entre la exigencia por producir y atender las necesidades del ámbito laboral y otras ocupaciones, y colocaron al centro el autocuidado.

En este sentido, las dinámicas de trabajo se transformaron y tomaron un ritmo más lento para muchas de ellas, ya que se manifestó un agotamiento emocional: el trabajo realizado en casa implicó tanto el quehacer laboral como el doméstico, todo en un mismo espacio. A esto se le suma que el trabajo de atención de las redes sociodigitales exige mucho tiempo para las colectivas feministas: como consecuencia del confinamiento, se vieron en la necesidad de expandir sus redes y esto implicó más tiempo y labor en este periodo.

La transición del espacio físico al digital de las actividades de las colectivas feministas implicó que, en algunos casos, necesitaran pagar para tener apoyo en el uso de las herramientas digitales, por ejemplo, en las transmisiones en vivo y como complemento de lo que aprendían a hacer de manera personal. Por otro lado, el tránsito hacia lo digital conllevó una resistencia al cambio de las actividades presenciales al espacio digital, ya que las dinámicas en lo cotidiano implicaban reunirse con las mujeres a diario, trabajar desde la colectividad, sentir el espacio seguro y llevar a cabo conferencias presenciales. No compartir en el espacio físico fue difícil, dado que la dinámica de trabajo ocurría todo el tiempo por la plataforma Zoom, y eso supone un agotamiento extremo.

Las activistas entrevistadas consideraron que a partir de la marcha del \#8M, realizada el 8 de marzo de 2020, se advirtió una de las ventajas de la transición de lo físico a lo digital, ya que ello impactó en la visibilidad de la problemática de violencia contra las mujeres en México. Ante la imposibilidad de tomar el espacio público presencialmente, mantienen la esperanza de que se sostendrá toda la organización emprendida por estas redes feministas, pues continúa la comunicación entre ellas, y todos estos factores, 
desde su perspectiva, hacen imparable el movimiento feminista en México. Sin embargo, coexiste una perspectiva crítica que considera que, a través de las redes sociodigitales como Twitter, las discusiones pueden ser «de escritorio» y no corresponderse con la realidad, dado que estar en la calle es lo que genuinamente ha potenciado al movimiento feminista en México.

No obstante, persiste un consenso en la idea de que tanto las acciones llevadas en línea como en la calle son formas válidas de protesta; a partir de los datos empíricos analizados, podemos indicar que no prevaleció una tensión entre los dos tipos de protesta. Incluso, a raíz de apropiarse del espacio digital, surgen nuevos retos con relación a la seguridad digital, dado el incremento de violencia digital contra las activistas feministas. Por ello, esta temática ha exigido implementar, dentro de las colectivas, estrategias de autocuidado, como, por ejemplo, mantener el anonimato, utilizar software libre que proporcione seguridad en sus interacciones digitales o en la comunicación al interior de la colectiva para detectar perfiles falsos o páginas de Facebook falsas, entre otras.

Consideramos que uno de los logros más transcendentales del movimiento feminista en México durante la pandemia por la COVID-19 ha sido la lucha por visibilizar, mediante las plataformas digitales, el incremento de la violencia contra las mu- jeres, ocasionado por el confinamiento de miles de mujeres con sus agresores, así como por la reducción del presupuesto económico y la falta de políticas públicas para atender este problema.

Las colectivas se apropiaron paulatinamente de herramientas digitales para tener actividad y producir sus contenidos por ese medio; lo hicieron, por ejemplo, al crear pódcast, infografías, campañas virtuales; transmitir conferencias vía streaming; o acompañar virtualmente a las víctimas. Ante la inacción de las instancias gubernamentales, el activismo de base liderado por mujeres en México ha ampliado rápidamente su alcance de múltiples maneras, reutilizando creativamente las actividades para ayudar a las comunidades a responder a la pandemia o exigiendo a los Gobiernos que rindan cuentas y actúen. Un ejemplo de esto fue el movimiento \#NosotrasTenemosOtrosDatos surgido durante la pandemia, que contrarrestó la narrativa del presidente Andrés Manuel López Obrador y la negación del incremento de llamadas al 911 por violencia doméstica.

A más de diecinueve meses del inicio del confinamiento por la pandemia generada por la COVID-19, uno de los principales retos que la agenda feminista debe considerar es este: ¿cómo incorporará todos los aprendizajes derivados de la protesta digital y los repertorios de acción colectiva en línea en defensa de las mujeres por una vida libre de violencia a la par con el 
retorno paulatino a la nueva normalidad, al espacio público y las calles? Asimismo, ¿qué tipo de medidas sanitarias tomarán para evitar contagios? ¿De qué manera puede impactar el activismo híbrido en la búsqueda de atención a sus demandas frente al Estado mexicano?

Por otro lado, el movimiento feminista en México enfrenta una estigmatización por parte de muchos medios de comunicación y de diversos actores políticos - desde el actual presidente de México- debido a la intervención de monumentos - denominada iconoclasia, que consiste en la destrucción de símbolos o monumentos con fines políticos o religiosos-. La protesta feminista ha tomado esta intervención como declaración legítima ante la inacción de las autoridades, las cuales ponen en tela de juicio diariamente los testimonios de miles de mujeres y niñas.

No obstante, este ha sido uno de los obstáculos para entablar un diálogo propositivo que dé paso a políticas públicas en favor de la erradicación de la violencia contra las mujeres. Por otro lado, esta emergencia nacional ha propiciado la conformación de nuevas colectivas feministas que atienden con sus propios recursos y acompañan a mujeres víctimas, lo que ha generado el fenómeno de la doble indignación al que se refiere Cerva Cerna (2020):

Frente a un acontecimiento de violencia -feminicidio, violencia sexual, desapariciones de mujeres-, la protesta emerge no sólo por el hecho en sí mismo, que en lo sustantivo se constituye en el primer agravio. El reclamo se extiende y profundiza a partir de un segundo momento de malestar colectivo que se produce por la forma en que las autoridades responden al primer agravio (p. 182).

En particular, la autora señala que la actual administración de Andrés Manuel López Obrador se ha caracterizado por la «reproducción de estereotipos con una fuerte carga de machismo» (Cerva Cerna, 2020, p. 193). Esto lo hemos podido confirmar con anterioridad en las conferencias matutinas del Poder Ejecutivo federal. Esta situación, lejos de ser un avance para combatir la violencia contra las mujeres y niñas, en el que prevalezca un puente de comunicación para la acción entre la sociedad civil y el Estado, agrava las problemáticas en un entorno de impunidad e indolencia por parte de las autoridades, y deja un panorama desalentador en el que las colectivas feministas deberán renovar sus repertorios de acción para ser escuchadas y concientizar a la ciudadanía mexicana de la violencia cotidiana a la que miles de mujeres están potencialmente expuestas.

El presente estudio se considera de tipo exploratorio, debido a la escasa literatura sobre las transformaciones en las prácticas comunicativas que han tenido las colectivas feministas como conse- 
cuencia de la pandemia por la COVID-19. Por tanto, este artículo aporta a la discusión elementos que pueden derivar en otras líneas de investigación para profundizar en responder las siguientes preguntas: ¿de qué manera se han modificado las prácticas comunicativas en los entornos digitales a partir del activismo feminista?, ¿cómo se han transformado las dinámicas comunicativas y de organización al interior de las colectivas feministas en el contexto de la pandemia por la COVID-19?, ¿qué implicaciones tiene el agotamiento debido al activismo feminista en los entornos digitales?, ¿qué estrategias han implementado las colectivas para contrarrestar los efectos del agotamiento derivados de su activismo feminista?, ¿qué retos tiene el movimiento feminista pospandemia COVID-19? 


\section{REFERENCIAS}

Al-Ali, N. (2020). Covid-19 and feminism in the Global South: Challenges, initiatives, and dilemmas. European Journal of Women's Studies, 27(4), 333-347. https://doi. org/10.1177/1350506820943617

Badillo, D. (2020, 23 de mayo). En México, los movimientos feministas siguen vivos: Wendy Figueroa. El Economista. https://www.eleconomista. com.mx/politica/En-Mexico-los-movimientos-feministas-siguen-vivos-Wendy-Figueroa-20200523-0018. $\underline{\mathrm{html}}$

Cerva Cerna, D. (2020). La protesta feminista en México. La misoginia en el discurso institucional y en las redes sociodigitales. Revista Mexicana de Ciencias Políticas y Sociales, 65(240),177205. http://dx.doi.org/10.22201/ fcpys.2448492xe.2020.240.76434

Della Porta, D. (2020a). How progressive social movements can save democracy in pandemic times. Interface: $A$ Journal for and About Social Movements, 12(1), 355-358. https://www. interfacejournal.net/wp-content/ uploads/2020/07/Interface-12-1-Della-Porta.pdf

Della Porta, D. (2020b). Movimientos sociales en tiempos de Covid-19: otro mundo es necesario. En B. Bringel y G. Pleyers (Eds.), Alerta global. Políticas, movimientos sociales y futuros en disputa en tiempos de pandemia (pp.175-180). CLACSO; ALAS. http:// biblioteca.clacso.edu.ar/clacso/ se/20200826014541/Alerta-global. pdf
El Gobierno de México está desarmando los mecanismos de protección a la mujer. (2020, 13 de julio). Mi Punto de Vista. http://www.mipuntodevista.com. $\mathrm{mx} /$ lel-gobierno-de-mexico-esta-desarmando-los-mecanismos-de-proteccion-a-la-mujer/?fbclid=IwAR1K Im6FhYeNod-pjvhEUJeiVnNCFZ-htJyNdDR8yclaL25PoyDWG 6UNY

James, N. y Busher, H. (2012). Internet interviewing. En J. F. Gubrium, J. A. Holstein, A. B. Marvasti y K. D. McKinney (Eds.), The SAGE handbook of interview research: The complexity of the craft (2. ${ }^{\mathrm{a}}$ ed.) (pp. 177-191). SAGE Publications.

Klandermans, B. y Staggenborg, S. (Eds.). (2002). Methods of social movement research. University of Minnesota Press.

Mendes, K., Ringrose, J. y Keller, J. (2019). Digital feminist activism: Girls and women fight back against rape culture. Oxford University Press. https://doi.org/10.1093/ oso/9780190697846.001.0001

Núñez Cetina, S. (2021). Violencia contra las mujeres y feminicidio íntimo a la sombra del covid-19. Los efectos perversos del confinamiento. Política y Cultura, (55), 99-119. https://polcul. xoc.uam.mx/index.php/polcul/article/view/1451/1407 
Observatorio Ciudadano Nacional del Feminicidio. (2021, 23 de febrero). Violencia contra las mujeres en México. Informe del OCNF, CDD y REDTDT al Comité CEDAW. https://www. observatoriofeminicidiomexico. org/post/violencia-contra-las-mujeres-en-m $\% \mathrm{C}_{3} \% \mathrm{~A} 9 \times \mathrm{xico}$-informe-del-ocnf-cdd-y-redtdt-al-comit\% $3 \%$ A9-cedaw?fbclid=IwAR3 uizkpGPg9vAAoabP 5 upH $_{5}$ YR hEADvxUzSTRIngFM4kgf2Ly6gNaiNlM

Piñeiro-Otero, T. (2021). «Escúchanos, hermana». Los podcast como prácticas y canales del activismo feminista. Revista Inclusiones, 8(Especial), 231254. https://revistainclusiones.org/ index.php/inclu/article/view/2600

Pleyers, G. (2020). The pandemic is a battlefield. Social movements in the COVID-19 lockdown, Journal of Civil Society, 16(4), 295-312. https://doi.or $\mathrm{g} / 10.1080 / 17448689.2020 .1794398$

Pleyers, G. (2021). Movimientos sociales y ayuda mutua frente a la pandemia. Mundos Plurales. Revista Latinoamericana de Políticas y Acción Pública, 8(1), 9-22. https://doi.org/10.17141/ mundosplurales.2.2020.4873.

Portillo Sánchez, M. y Beltrán Fuentes, D. (2020). Efectos de la pandemia por la Covid-19 en las movilizaciones feministas de la Ciudad de México. Revista Mexicana de Estudios de los Movimientos Sociales, 5(1), 6-36. http://www.revistamovimientos.mx/ ojs/index.php/movimientos/article/ view/250.

Ragin, C. C. y Amoroso, L. M. (1994). Constructing social research: The unity and diversity of method. SAGE Publications.
Registro Nacional de Personas Desaparecidas y No Localizadas. (2021). Versión pública RNPDNO. https://versionpublicarnpdno.segob.gob.mx/Dashboard/ContextoGeneral

Restauradoras con Glitter. (2019, diciembre). Nosotras. https://restauradorasconglitter.com/nosotras/

Rodriguez, L. (2021, 30 de junio). How has the COVID-19 pandemic impacted feminist movements? Global Citizen. https://www.globalcitizen.org/ en/content/covid-19-feminist-movements-generation-equality/?template $=$ next

Secretariado Ejecutivo del Sistema Nacional de Seguridad Pública. (2021, 20 de enero). Incidencia delictiva del fuero común 2020. Instrumento para el registro, clasificación y reporte de delitos y las víctimas. CNSP/38/25. Centro Nacional de Información. https:// drive.google.com/file/d/1IAsT34UrJ85b4z8RkQGYlt8yU5corMPS/view

Secretariado Ejecutivo del Sistema Nacional de Seguridad Pública. (2021a, 31 de marzo). Información sobre violencia contra las mujeres. Incidencia delictiva y llamadas de emergencia 9-1-1. Centro Nacional de Información. https://drive.google.com/file/d/1IFK FRGveCmvgeCWSlHJ7s 5u2DHw3N7/view

Secretariado Ejecutivo del Sistema Nacional de Seguridad Pública. (2021b, 30 de abril). Información sobre violencia contra las mujeres. Incidencia delictiva y llamadas de emergencia 9-1-1. Centro Nacional de Información. https://drive.google.com/file/d/16iSIf8WBmnlrUEsaKWyey7 mJt2AMFSE/view 
Suárez-Krabbe, J. (2020). [COVID-19 pandemic: Worlds stories from the margins] Relinking as healing: Ruminations on crises and the radical transformation of an antisocial and antirelational world. Convivial Thinking, https:// www.convivialthinking.org/index. $\mathrm{php} / 2020 / 10 / 05 /$ relinking-as-healing/

Tabbush, C. (2021). La pandemia, una encrucijada para la igualdad de género. Nueva Sociedad, (293), 93-105. https://static.nuso.org/media/articles/ downloads/5.TC Tabbush 293.pdf

Tabbush, C. y Friedman, E. J. (2020). Feminist activism confronts COVID-19. Feminist Studies, 46(3), 629-638. https://doi.org/10.15767/feministstu$\underline{\text { dies.46.3.0629 }}$

Ventura Alfaro, M. J. (2020). Feminist solidarity networks have multiplied since the COVID-19 outbreak in Mexico. Interface: A Journal for and About Social Movements, 12(1), 82-87. https://www.interfacejournal.net/ wp-content/uploads/2020/o7/Interface-12-1-Ventura-Alfaro.pdf

Xantomila, J. (2020, 1 de noviembre). Mujeres participan en Marcha de las Catrinas, contra feminicidios. La Jornada. https://www.jornada.com. $\mathrm{mx} /$ ultimas/sociedad/2020/11/01/ mujeres-participan-en-marcha-de-las-catrinas-contra-feminicidios-450.html 